Acta Crystallographica Section D

Biological

Crystallography

ISSN 0907-4449

Scott D. Pegan, ${ }^{\mathrm{a} *}$ Hector Serrano, ${ }^{\text {b }}$ Christian P. Whitman ${ }^{\text {b }}$ and Andrew D. Mesecar ${ }^{\mathrm{a} *}$

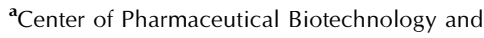
the Department of Medicinal Chemistry and Pharmacognosy, University of Illinois, Chicago, USA, and ${ }^{\mathbf{b}}$ Division of Medicinal Chemistry, College of Pharmacy, The University of Texas, Austin, USA

Correspondence e-mail: pegan@uic.edu, mesecar@uic.edu

\title{
Structural and mechanistic analysis of trans-3-chloroacrylic acid dehalogenase activity
}

Trans-3-chloroacrylic acid dehalogenase (CaaD) is a critical enzyme in the trans-1,3-dichloropropene (DCP) degradation pathway in Pseudomonas pavonaceae 170. This enzyme allows bacteria to use trans-DCP, a common component in commercially produced fumigants, as a carbon source. CaaD specifically catalyzes the fourth step of the pathway by cofactorindependent dehalogenation of a vinyl carbon-halogen bond. Previous studies have reported an X-ray structure of CaaD under acidic conditions with a covalent modification of the catalytic $\beta$ Pro1 residue. Here, the $1.7 \AA$ resolution X-ray structure of CaaD under neutral $(\mathrm{pH}$ 6.5) conditions is reported without the presence of the covalent adduct. In this new structure, a substrate-like acetate molecule is bound within the active site in a position analogous to the putative substrate-binding site. Additionally, a catalytically important water molecule was identified, consistent with previously proposed reaction schemes. Finally, flexibility of the catalytically relevant side chain $\alpha$ Glu52 is observed in the structure, supporting its role in the catalytic mechanism.

\section{Introduction}

The 1,3-dichloropropene (DCP) degradation pathway facilitates the use of DCP as a carbon source for various soil bacteria including Pseudomonas pavonaceae 170 and coryneform bacterium strain FG41 (Fig. 1; Poelarends et al., 2001). DCP is a mixture of trans and cis isomers and is the active component of the commercially available fumigants Telone II and Shell D-D which are used as pesticides against plant parasitic nematodes. The DCP-degradation pathway and other dehalogenase pathways are of interest owing to their potential industrial use (Janssen et al., 2001). The DCP pathway is comprised of five enzyme-mediated steps (Fig. 1). The initial step requires a haloalkane dehalogenase to liberate the halogen from the $s p^{3} \mathrm{C}$ atom of DCP, eventually replacing it with a water-derived hydroxyl group. The 1-chloro-3-hydroxypropene product undergoes two sequential oxidation steps to yield trans- or cis-3-chloroacrylic acid. Subsequently, cis-3chloroacrylic acid dehalogenase (cis-CaaD) and trans-3chloroacrylic acid dehalogenase (CaaD) mediate the release of the halogen from the $s p^{2} \mathrm{C}$ atom to yield malonate semialdehyde and $\mathrm{HCl}$. This product is then decarboxylated by malonate semialdehyde decarboxylate, producing acetaldehyde, which is then likely to be funneled to the Krebs cycle (de Jong et al., 2004).

Of the several dehalogenases known to exist, cis-CaaD and $\mathrm{CaaD}$ are unique to the DCP-degradation pathway as they are the only known dehalogenases that are capable of cleaving vinyl carbon-halogen bonds without the assistance of co-
Received 24 September 2008 Accepted 23 October 2008

PDB References: CaaD, wild type, 3ej3, r3ej3sf; $\alpha \mathrm{E} 52 \mathrm{Q}$, 3ej7, r3ej7sf; $\alpha$ R8A, 3ej9, r3ej9sf. 
factors (Poelarends et al., 2001). In addition, these isomerspecific dehalogenases do not belong to the $\alpha / \beta$-hydrolase short-chain dehydrogenase/reductase (SDR) or the haloacid dehalogenase (HAD) superfamilies (de Jong \& Dijkstra, 2003). Previous studies have placed them in the tautomerase superfamily (de Jong et al., 2004, 2007; Poelarends \& Whitman, 2004). Tautomerase-superfamily members all contain at least one $\beta-\alpha-\beta$ structural motif and catalyze diverse chemical reactions using an $\mathrm{N}$-terminal proline as a catalytic residue (Whitman, 2002). Both dehalogenases exhibit these features, but their structural similarities end there. In contrast to cis$\mathrm{CaaD}$, which is a homotrimer comprised of 149 residues, CaaD is a heterohexamer composed of three heterodimers of $\alpha$ - and $\beta$-subunits that are 75 and 70 residues in length, respectively (de Jong et al., 2004, 2007).

Based on sequence similarity to 4-oxalocrotonate tautomerase (4-OT) and mutagenesis experiments, it was initially proposed that $\beta$ Pro1 of CaaD functions as a base to activate water for addition to $\mathrm{C}-3$ of the substrate and that $\alpha \mathrm{Arg} 11$ interacts with the $\mathrm{C}-1$ carboxylate group and functions as an electron sink (Poelarends et al., 2001). Mutagenesis showed that both residues were required for activity. The observed hydration reaction also suggested that 3 -bromopropiolate and 3-chloropropiolate could be transformed into irreversible inhibitors of CaaD through modification of an active-site residue (Wang et al., 2003). It was subsequently found that both compounds inactivate CaaD by modification of $\beta$ Pro1 with 3-oxopropanoate, the species resulting from hydration of the 3-halopropiolates (Wang et al., 2003).

Structural work, coupled with mutagenesis and determination of the $\mathrm{p} K_{\mathrm{a}}$ value for $\beta$ Pro1, more precisely elucidated the catalytic mechanism of CaaD. A crystal structure of CaaD in which $\beta$ Pro1 is modified by 3-oxopropanoate showed the positions of $\beta$ Pro1, $\alpha \operatorname{Arg} 8, \alpha \operatorname{Arg} 11$ and $\alpha$ Glu52 relative to the

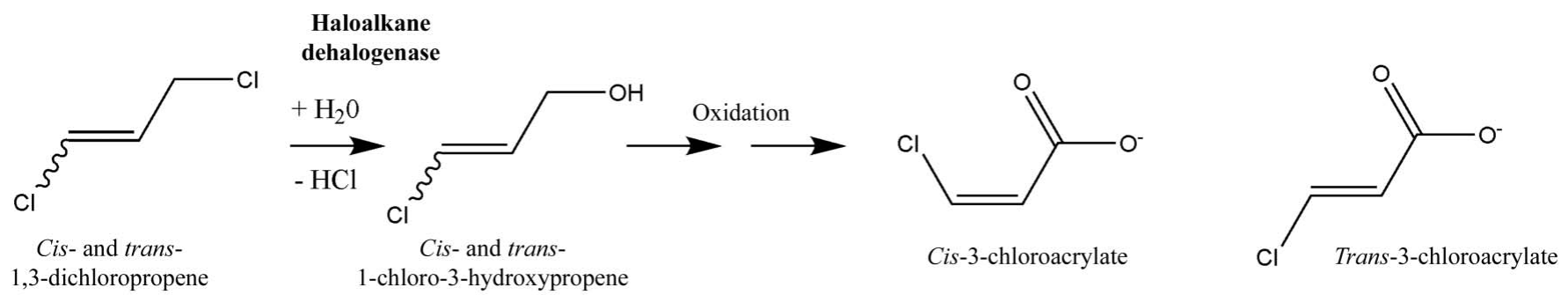

Cis- and trans-
1,3-dichloropropene

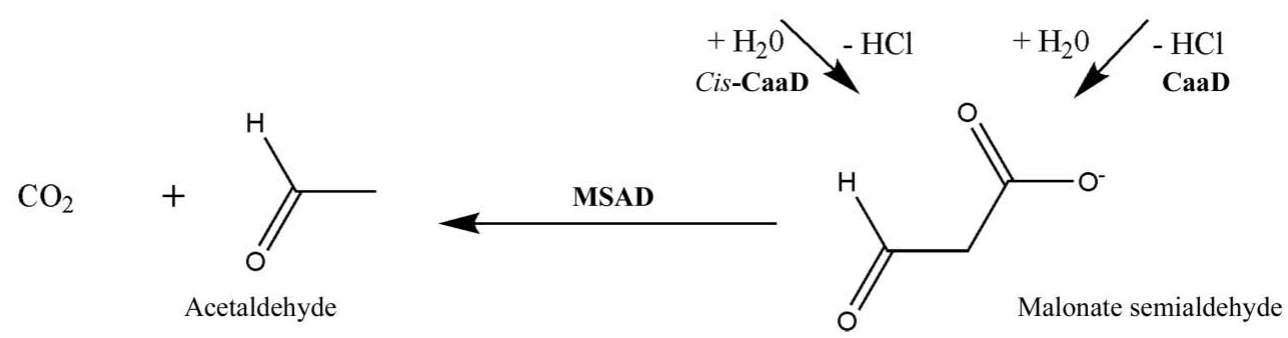

Figure 1

The DCP-degradation pathway in P. pavonaceae 170 and coryneform bacterium strain FG41. CaaD and cis-CaaD refer to trans- and cis-3-chloroacrylic acid dehalogenase, respectively. MSAD refers to malonate semialdehyde decarboxylase.

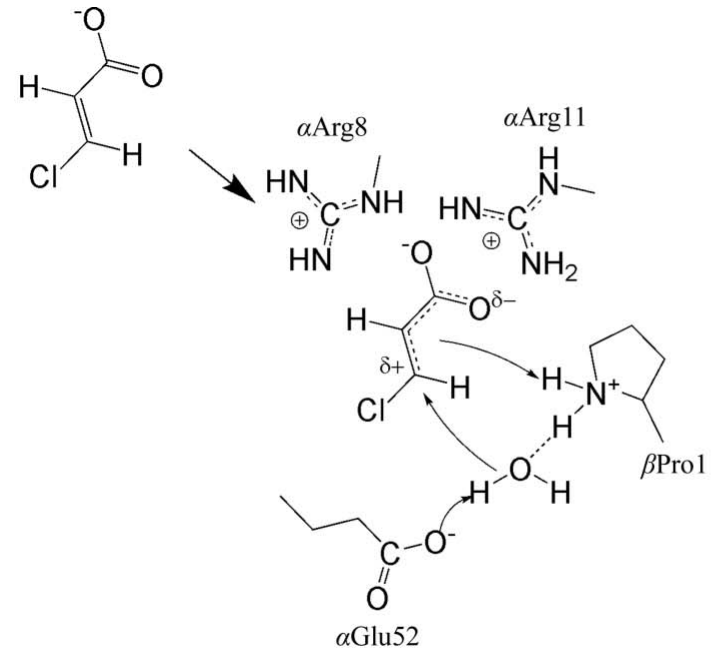

Figure 2

Proposed $\mathrm{CaaD}$ reaction scheme.

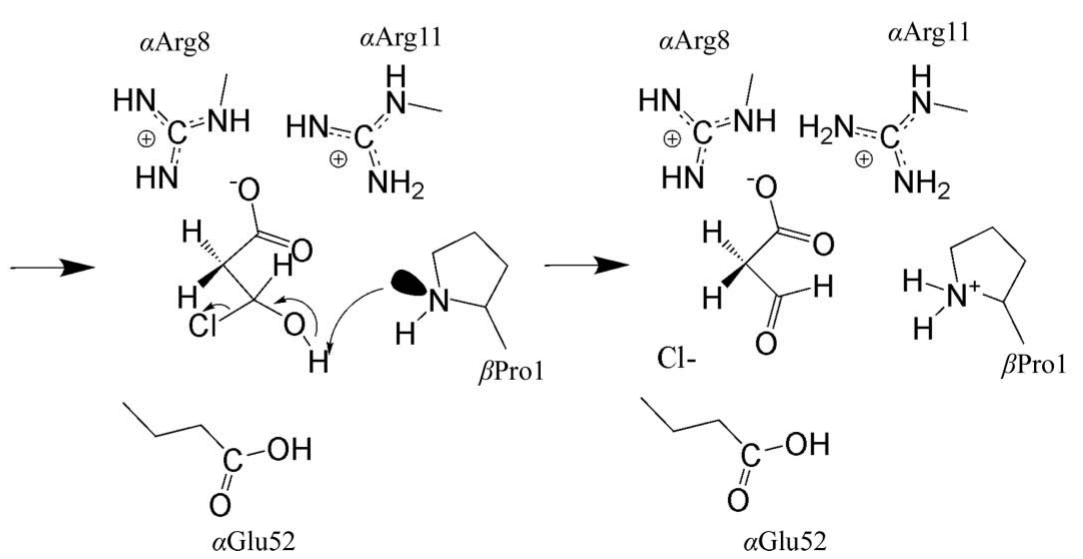


covalent adduct (de Jong et al., 2004). Extrapolation of these observations to substrate turnover suggested that $\alpha$ Glu52 functions as a general base to activate a water molecule, whereas $\beta$ Pro1 completes the addition of water by placing a proton at C-2 (Fig. 2). The pair of arginines interacts with the carboxylate group and serves two major purposes: it holds the substrate in place and polarizes the $\alpha, \beta$-unsaturated acid. The results of mutagenesis experiments implicated these residues as being critical for activity. Finally, the $\mathrm{p} K_{\mathrm{a}}$ of $\beta$ Pro1 was determined to be $\sim 9.2$ and is consistent with its role as a general acid (Azurmendi et al., 2004).

The aforementioned studies, sequence analysis and subsequent crystallographic work formed the basis for the mechanism of cis-CaaD. The active-site players in the cisCaaD mechanism are Pro1, Glu114, Arg70 and Arg73 (Poelarends et al., 2004). Two additional residues, His28 and Tyr103, were uncovered in the crystallographic work performed on cis-CaaD (de Jong et al., 2007). Although the CaaD and cis-CaaD structures were important for identifying the active-site residues important for binding and catalysis, none of the structures were able to confirm the presence of a catalytically important water molecule proposed to be essential for the reaction scheme. Moreover, the structures of the covalently modified $\mathrm{CaaD}$ and cis-CaaD may be deceptive in that the resulting adducts may not reflect the true catalytic state of the active site. For example, the observed hydrogen bonds in the covalently modified enzymes may not be present in the native enzymes. Thus, the respective adducts may obscure additional structural information that is essential for a more complete understanding of the $\mathrm{CaaD}$ and cis-CaaD reaction mechanisms. In addition, all of the previous structures were crystallized under $\mathrm{pH}$ conditions that are well below the optimal $\mathrm{pH}$ of the enzyme.

To provide further details of the $\mathrm{CaaD}$ reaction mechanism, we elucidated a high-resolution noncovalently modified $\mathrm{CaaD}$ structure with a substrate-like acetate molecule trapped in the active site. In addition, the X-ray structures of two $\mathrm{CaaD}$ mutants, $\alpha$ E52Q CaaD and $\alpha \mathrm{R} 8 \mathrm{~A} \mathrm{CaaD}$, were also determined. The unmodified $\mathrm{CaaD}$ structure was determined under neutral $\mathrm{pH}$ conditions and allowed us to identify the location of the catalytic water that was hypothesized to exist in previous proposed mechanisms. Finally, we also observe inherent flexibility in the catalytically relevant side chain of $\alpha$ Glu52. Together, the structural observations furnish further and new mechanistic details of the CaaD reaction mechanism.

\section{Methods and materials}

Wild-type (wt) CaaD and the $\alpha \mathrm{E} 52 \mathrm{Q}$ and $\alpha \mathrm{R} 8 \mathrm{~A}$ mutants were expressed and purified according to previously described methodology (Poelarends et al., 2001; Wang et al., 2003). During the final protein preparation for crystallization, wt $\mathrm{CaaD}$ and the $\alpha \mathrm{E} 52 \mathrm{Q}$ and $\alpha \mathrm{R} 8 \mathrm{~A}$ mutants were concentrated to 12,13 and $10 \mathrm{mg} \mathrm{ml}^{-1}$, respectively, in $10 \mathrm{~m} M$ sodium phosphate buffer $\mathrm{pH}$ 8.0. The final enzyme concentrates were stored at $277 \mathrm{~K}$. Initial crystallization conditions for the three $\mathrm{CaaD}$ proteins were determined from high-throughput
Table 1

Data-collection and refinement statistics.

Values in parentheses are for the last resolution shell.

\begin{tabular}{|c|c|c|c|}
\hline & Wild type & $\alpha \mathrm{R} 8 \mathrm{~A}$ & $\alpha \mathrm{E} 52 \mathrm{Q}$ \\
\hline \multicolumn{4}{|l|}{ Data collection } \\
\hline Space group & $P 2_{1}$ & $P 2_{1} 2_{1} 2_{1}$ & $P 2_{1} 2_{1} 2_{1}$ \\
\hline \multicolumn{4}{|l|}{ Unit-cell parameters } \\
\hline$a(\AA)$ & 50.7 & 60.3 & 56.3 \\
\hline$b(\AA)$ & 97.3 & 83.6 & 69.7 \\
\hline$c(\AA)$ & 69.0 & 124.1 & 89.9 \\
\hline$\alpha\left(^{\circ}\right)$ & 90.0 & 90.0 & 90.0 \\
\hline$\beta\left(^{\circ}\right)$ & 96.1 & 90.0 & 90.0 \\
\hline$\gamma\left({ }^{\circ}\right)$ & 90.0 & 90.0 & 90.0 \\
\hline Resolution $(\AA)$ & $68.7-1.7$ & $69.3-1.9$ & $55.1-1.5$ \\
\hline No. of reflections observed & 246877 & 359016 & 363433 \\
\hline No. of unique reflections & 70963 & 50414 & 56197 \\
\hline$R_{\text {merge }} \dagger(\%)$ & $5.6(26.0)$ & $8.9(48.3)$ & $9.8(36.8)$ \\
\hline$I / \sigma(I)$ & $37.4(3.8)$ & $23.0(2.75)$ & $18.4(5.1)$ \\
\hline Completeness (\%) & $96.8(82.4)$ & $98.9(95.2)$ & $97.4(86.1)$ \\
\hline \multicolumn{4}{|l|}{ Refinement } \\
\hline Resolution range & $68.7-1.7$ & $69.3-1.9$ & $55.1-1.5$ \\
\hline No. of reflections in working set & 67363 & 47330 & 53285 \\
\hline No. of reflections in test set & 3599 & 2544 & 2850 \\
\hline$R_{\mathrm{work}} \ddagger(\%)$ & 17.9 & 20.0 & 21.5 \\
\hline$R_{\text {free }} \neq(\%)$ & 21.8 & 26.8 & 25.1 \\
\hline Average $B$ factor $\left(\AA^{2}\right)$ & 25 & 15.8 & 27 \\
\hline \multicolumn{4}{|l|}{ R.m.s. deviations } \\
\hline Bond lengths $(\AA)$ & 0.013 & 0.014 & 0.01 \\
\hline Bond angles $\left({ }^{\circ}\right)$ & 1.45 & 1.41 & 1.10 \\
\hline Protein/water atoms & $5818 / 560$ & $5827 / 554$ & $2902 / 378$ \\
\hline Hexamers in ASU & 2 & 2 & 1 \\
\hline
\end{tabular}

$\dagger R_{\text {merge }}=\sum_{h k l} \sum_{i}\left|I_{i}(h k l)-\langle I(h k l)\rangle\right| / \sum_{h k l} \sum_{i} I_{i}(h k l)$, where $I_{i}(h k l)$ is the $i$ th measurement and $\langle I(h k l)\rangle$ is the weighted mean of all measurements of $I(h k l)$. $\quad \ddagger R_{\text {work }}$ and $R_{\text {free }}=h\left[\left|F(h)_{\text {obs }}\right|-\left|F(h)_{\text {calc }}\right|\right] / h\left|F(h)_{\text {obs }}\right|$ for reflections in the working and test sets, respectively.

screening of Hampton Research Index HT, Crystal Screen HT and SaltRx HT screens using a Tecan Freedom Evo 200 liquidhandling robot. The protein solution was mixed in a 1:1 ratio with precipitant solution to give a total volume of $2 \mu \mathrm{l}$ in a sitting drop that was formatted in microplates with $100 \mu \mathrm{l}$ reservoir solution. The final crystals of both wt $\mathrm{CaaD}$ and the $\alpha$ E52Q mutant were obtained by vapor diffusion using $4 \mu \mathrm{l}$ hanging drops over $500 \mu \mathrm{l}$ reservoir solution. Hanging drops were formed by mixing protein solution in a $1: 1$ ratio with precipitant solution, which consisted of $0.1 \mathrm{M}$ ammonium acetate, $0.1 M$ Bis-Tris buffer pH 6.5 and 25\% PEG 3350. The final crystals of the $\alpha \mathrm{R} 8 \mathrm{~A}$ mutant were obtained through similar methods except that $0.2 \mathrm{M} \mathrm{Li}_{2} \mathrm{SO}_{4}$ replaced $0.1 \mathrm{M}$ ammonium acetate in the precipitant.

$\mathrm{X}$-ray diffraction data were collected from wt CaaD crystals on SER-CAT beamline 22-BM and from the $\alpha$ R8A and $\alpha$ E52Q CaaD mutant crystals on SERCAT beamline 22-ID at the Advanced Photon Source (APS), Argonne National Laboratory. Crystals were mounted on nylon loops and submerged in a $4 \mu \mathrm{l}$ drop of well solution. The crystals were immediately flash-frozen by submerging them in liquid nitrogen. The flash-frozen crystals were mounted on a goniostat under a stream of dry $\mathrm{N}_{2}$ at $100 \mathrm{~K}$. X-ray exposures of $5 \mathrm{~s}$ per degree of rotation over a total of $200^{\circ}$ in $\omega$ were collected on a MAR $225 \mathrm{CCD}$ detector for wt $\mathrm{CaaD}$ and on a MAR 300 CCD detector for crystals of the CaaD mutants. $\mathrm{X}$-ray data were processed and scaled using $H K L-2000$ 
(Otwinowski \& Minor, 1997) and the statistics are presented in Table 1. Molecular-replacement solutions were derived with Phaser using the covalently modified CaaD structure (PDB code $1 \mathrm{~s} 0 \mathrm{y})$ as a search model for the wt CaaD structure. The final refined structure of wt $\mathrm{CaaD}$ was used as a search model for the $\alpha \mathrm{E} 52 \mathrm{Q}$ and $\alpha \mathrm{R} 8 \mathrm{~A}$ mutant structures. WinCoot was used for model building and REFMAC from the CCP4 software suite was used for structural refinement (Collaborative Computational Project, Number 4, 1994; Emsley \& Cowtan, 2004).

\section{Results and discussion}

To obtain a noncovalently modified $\mathrm{CaaD}$ structure, wt $\mathrm{CaaD}$ was screened against 384 precipitant conditions from Hampton Research HT screens. Numerous thin plate-like crystals with a morphology described in previous studies were observed after $2 \mathrm{~d}$ incubation at room temperature (de Jong et al., 2004). Crystals originating from these drops produced multiple lattice diffraction images. However, thick homogeneous hexagonal plates were grown under a few conditions. Ultimately, optimization of crystal growth through various gradient hanging-drop strategies produced wt CaaD crystals that diffracted to $1.7 \AA$ resolution. The X-ray structure was subsequently solved by molecular replacement using the covalently modified $\mathrm{CaaD}$ structure (PDB code $1 \mathrm{~s} 0 \mathrm{y}$ ) as a search model (de Jong et al., 2004). CaaD crystallized in space group $P 2_{1}$ with two heterohexamers in the asymmetric unit (Table 1).
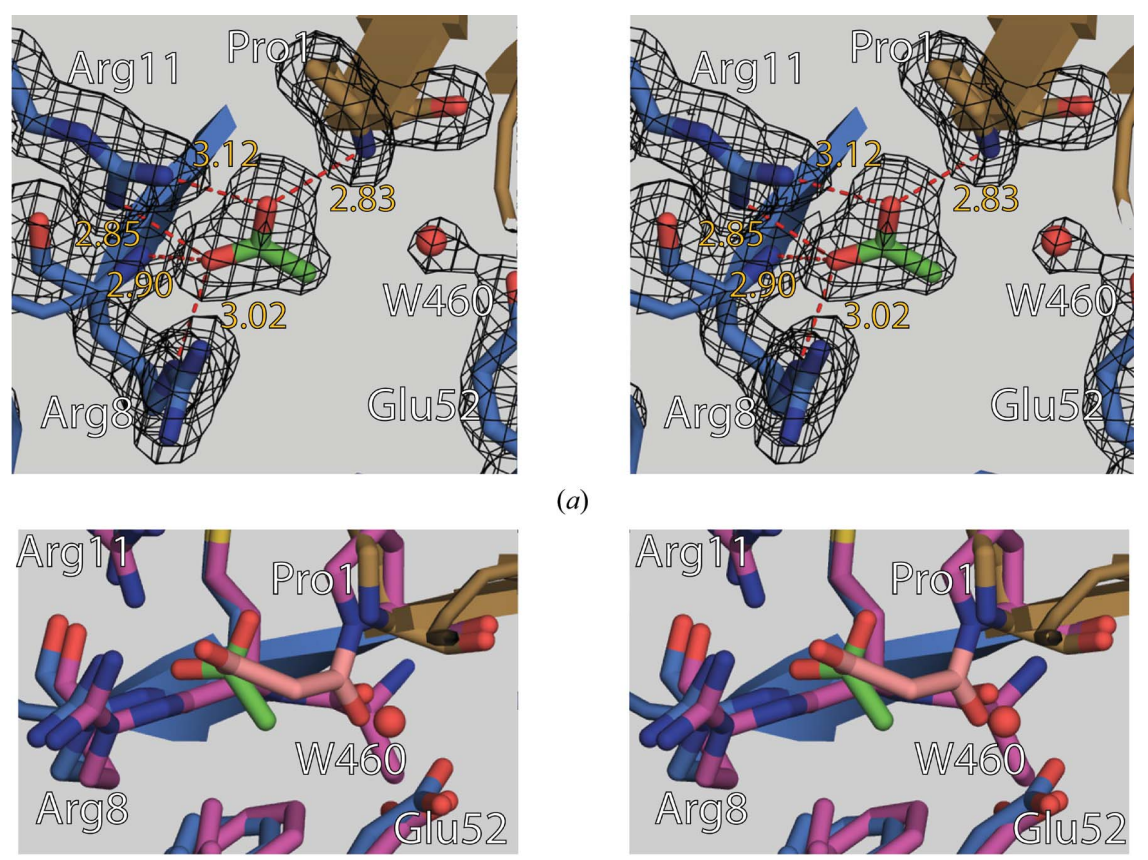

(a)

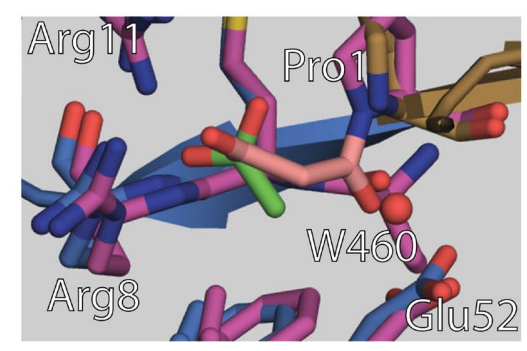

(b)

Figure 3

Active site of wt CaaD bound with acetate. (a) Divergent-eyed stereoview of the CaaD active site formed by $\alpha$ subunit $G$ (marine) and $\beta$-subunit $J$ (gold) with the acetate molecule in green. Water molecules are depicted as red spheres and the distances $(\AA)$ are shown as dashed lines (red). The $2 F_{\mathrm{o}}-F_{\mathrm{c}}$ electron-density maps (black) are contoured at 1.0 $\sigma$. (b) Divergent-eyed stereoview of the CaaD active site ( $a$ ) with $\beta$ Pro1 modified by 3-oxopropanoate (PDB code $1 \mathrm{~s} 0 \mathrm{y}$; magenta) superimposed. The covalent adduct is highlighted in pink.
Overall, the X-ray structure of wt CaaD closely resembles that of the 3-oxopropanoate-modified CaaD structure in terms of both secondary structure and quaternary structure. Close inspection of all six of the $\beta$ Pro1 active sites located in the asymmetric unit (three active sites per heterohexamer) revealed a significant peak in $F_{\mathrm{o}}-F_{\mathrm{c}}$ electron-density difference maps within the active site. Modelling of water at this location failed to accurately account for the density. Only acetate, a component of the precipitant, completely accounted for the density (Fig. 3a). All six actives sites had the acetate molecule present, which was positioned in the sites to allow the carboxylate group to form hydrogen-bonding interactions ranging from 2.8 to $3.1 \AA$ with $\alpha \operatorname{Arg} 8, \alpha \operatorname{Arg} 11$ and $\beta$ Pro1, suggesting a substrate-like interaction mimicking the carboxylic acid found in trans-3-chloroacrylic acid (Fig. 3a). Interestingly, the acetate molecule is not completely coplanar with $\alpha \operatorname{Arg} 11$. The acetate molecule forms hydrogen bonds to the secondary amine of the $\alpha \operatorname{Arg} 8$ side chain and the N-terminal amine of $\beta$ Pro1, resulting in a $12^{\circ}$ twist. This hydrogen-bond formation by the active-site arginines with the acetate may contribute to the formation of crystals for wt CaaD.

The $\alpha \operatorname{Arg} 8$ mutant failed to crystallize in the same precipitant as wt CaaD. Furthermore, attempts to crystallize an $\alpha$ R11A CaaD mutant failed in all of the 384 conditions attempted. However, the X-ray structure of $\alpha$ R8A was obtained from crystals grown from a similar precipitant with $0.2 M \mathrm{LiSO}_{4}$ replacing $0.1 M$ sodium acetate in the precipitant (Table 1). The replacement of acetate with the sulfate anion prevented the possible visualization of acetate in the active site of this mutant. As the active sites of $\mathrm{CaaD}$ and cis-CaaD have numerous positively charged side-chain residues, sulfate ions have been observed previously in the active site of cisCaaD (de Jong et al., 2007). However, no sulfate or other negatively charged ions were observed in the active site of the $\alpha$ R8A CaaD mutant (data not shown). With exception of the mutation of arginine to alanine, the active site of the $\alpha \mathrm{R} 8 \mathrm{~A} \mathrm{CaaD}$ otherwise resembles that of wt CaaD. This suggests that the reduced activity of this mutant observed in previous studies is solely a consquence of the removal of the contribution of the arginine to binding the substrate as well as its partial role as an electron sink (Poelarends et al., 2001).

Superposition of the covalently modified $\mathrm{CaaD}$ structure and the wt $\mathrm{CaaD}$ structure highlights the structural changes induced by the presence of the 3-oxopropanoate adduct at $\beta$ Pro1 in the covalently modified CaaD structure (Fig. $3 b$ ). In the native $\mathrm{CaaD}$ structure, $\alpha \operatorname{Arg} 8$ and $\alpha \operatorname{Arg} 11$ prefer to form bonds to the carboxylic acid moiety. In the covalently modified CaaD structure, these 
arginines form bonds to the carboxylic acid group of the 3oxopropanoate adduct. To accommodate this interaction, the modified $\beta$ Pro1 rotates out of plane compared with the wt $\mathrm{CaaD}$ structure (Fig. $3 b$ ). Unlike other families of dehalogenase enzymes that form covalent intermediates during their reaction schemes, a covalent intermediate is not present in the $\mathrm{CaaD}$ mechanism, thus suggesting that the proline in the wt structure is in its natural conformation.

In addition to inducing a rotation of $\beta$ Pro1, the keto $\mathrm{O}$ atom of the adduct contributes to hydrogen-bond interactions that stabilize the side chain of $\alpha$ Glu52. In the covalently modified CaaD structure, in which two heterohexamers are present in the asymmetric unit, all six $\alpha$ Glu52 side chains have well defined $2 F_{\mathrm{o}}-1 F_{\mathrm{c}}$ density at $1 \sigma$ (de Jong et al., 2004). However, in the wt structure only three of the six $\alpha$ Glu52 side chains present in the two heterohexamers in the asymmetric unit have well defined density in one conformation. The side chain of $\alpha$ Glu52 in this conformation, designated $\alpha$ Glu52c1,

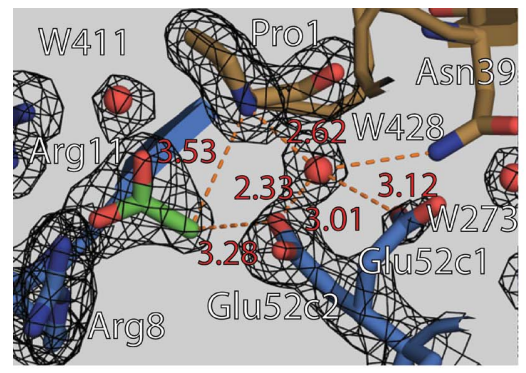

(a)
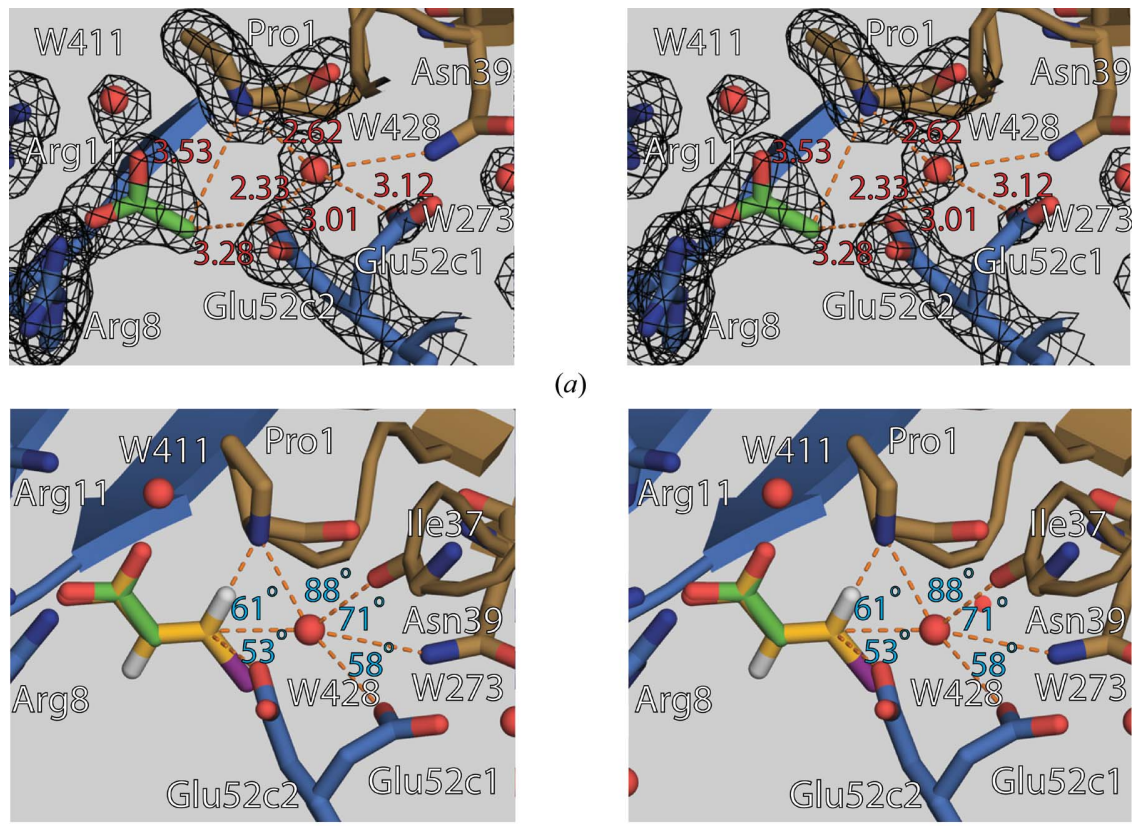

(b)
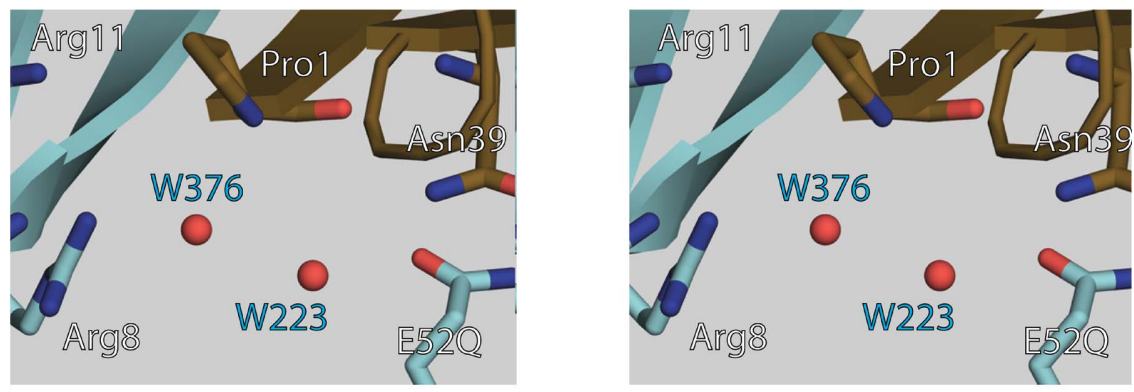

(c)

\section{Figure 4}

Role of $\alpha$ Glu52 and activated water W428 in CaaD catalysis. (a) Divergent-eyed stereoview of the $\mathrm{CaaD}$ active site formed by $\alpha$-subunit $A$ (marine) and $\beta$-subunit $F$ (gold) with the acetate molecule shown in green. Water molecules are depicted as red spheres and the distances $(\AA)$ as dashed lines (orange). The $2 F_{\mathrm{o}}-F_{\mathrm{c}}$ electron-density maps (black) are contoured at $1.0 \sigma .(b)$ Divergent-eyed stereoview of the CaaD active site in $(a)$ with trans-3-chloroacrylic acid (gold) superimposed on the acetate. Angles are shown as lines in orange with labels depicted in cyan. (c) Divergent-eyed stereoview of the active site of the $\alpha \mathrm{E} 52 \mathrm{Q}$ mutant of CaaD formed by $\alpha$-subunit $A$ (cyan) and $\beta$-subunit $F$ (dark gold). forms hydrogen bonds to the side-chain amide group of $\beta$ Asn39 and the backbone carboxyl group of $\alpha$ Ile 37 , similar to those observed in the modified CaaD structure (Figs. $3 b$ and 4). However, the absence of the keto oxygen in the adduct alters the remaining hydrogen-bonding partners to two waters, allowing the side chain of $\alpha$ Glu52 to move closer to the $\beta$ Asn39 side chain. In addition, one $\alpha$ Glu52, that of chain $A$, is observed to adopt two conformations when the occupancy of the atoms is set to be equal. The second conformation of $\alpha$ Glu52, designated $\alpha$ Glu52c2, involves side-chain rotation which results in partial blocking of substrate access to the active site. In this position, the $\alpha$ Glu52 side chain only forms en-bonding interactions with water 428 (Fig. $4 a$ ) ability of the side chain to move into the second conformation may facilitate the movement of water molecules to carry out the proposed hydration step of the dehalogenase reaction mechanism. When positioned in the first conformation, the side chain of $\alpha$ Glu52 creates a barrier between water 428 and the solvent-accessible water 273 (Fig. 4b). In the same conformation, $\alpha$ Glu52 is positioned optimally to activate water 428 as well as facilitate substrate uptake and product release.

We used the bound acetate molecule as an anchor point to model the CaaD substrate trans-3-chloroacrylic acid into the site. The resulting distance between water molecule W428 and C-3 of trans-3-chloroacrylic acid is $2.5 \AA$ (Fig. 4b). This distance is ideal for a reaction between the double bond of the substrate and a nearby water molecule (W428). Additionally, W428 forms hydrogen bonds to $\beta$ Pro1, $\alpha$ Glu52, $\beta$ Asn 39 and the carbonyl group of $\beta$ Ile 37 , placing the water in the correct orientation to be an activated water molecule and to perform a nucleophilic attack on the acetate substrate analog. The orientation of W428 in the unmodified active site and its proximity to $\mathrm{C}-3$ of the modelled trans-3-chloroacrylic acid suggest that W428 represents the catalytic water for this particular active site. Of the six active sites present in the asymmetric unit, three contain difference electron density that supports water at this position in the site and the inclusion of W460 in the active site formed from subunits $G$ and $J$ (Fig. 2). Once this water has been utilized by the hydration step of the proposed mechanism, the only way to replace the water is by $\alpha$ Glu52 adopting conformation 2, which can also support hydrogen bonding to that site (Figs. 2 and $3 a$ ). These waters were likely not 
to be present in the previous $\mathrm{CaaD}$ structures because these structures were determined under acidic conditions and $\beta$ Pro 1 prevented the formation of the hydrogen bond between it and the catalytic water. Under the previous acidic conditions used to crystallize $\mathrm{CaaD}, \alpha \mathrm{Glu} 52$ is expected to be protonated, thereby preventing the required hydrogen bonding necessary to stabilize the catalytic water and potentially explaining its absence.

The importance of this specific hydrogen-bonding pattern is further supported by the structure of the $\alpha \mathrm{E} 52 \mathrm{Q}$ mutant of CaaD. This mutant crystallized in the same acetate-containing precipitant as the native $\mathrm{CaaD}$ with one heterohexamer in the asymmetric unit. However, no acetate was located in the active site (Table 1, Fig. $4 c$ ). In the active site of the $\alpha \mathrm{E} 52 \mathrm{Q}$ mutant, $\alpha \mathrm{Gln} 52$ forms a stable polar interaction with $\beta$ Asn 39 in all three active sites present in the asymmetric unit (Fig. 4c). In addition to glutamine being a poor base, its interaction with $\beta$ Asn39 appears to lock the glutamine into a conformation that prevents it from adopting a conformation that would allow coordination of the catalytic water. The lack of the catalytic water disrupts the hydrogen-bond coordination of the $\beta$ Pro1 $\mathrm{N}$-terminal $\mathrm{N}$ atom, a hydrogen-bonding partner of the bound acetate in native CaaD. With the exception of the interaction between the glutamate and $\beta$ Asn 39 , the active site of the $\alpha \mathrm{E} 52 \mathrm{Q}$ mutant otherwise closely resembles that of wt $\mathrm{CaaD}$. As a result, the lack of activity observed previously in the $\alpha \mathrm{E} 52 \mathrm{Q}$ mutant enzyme can be directly attributed to the loss of the catalytic base and the inability to position a water molecule (Poelarends et al., 2001).

\section{Conclusion}

Elucidation of the wt CaaD structure provides further insight into the reaction mechanism of this class of dehalogenase enzyme. Observed in the active site was an acetate molecule that is thought to mimic the carboxylic acid of its substrate trans-3-chloroacrylic acid, suggesting that the noncovalently modified structure resembles a partial substrate-bound state consistent with previous studies (Wang et al., 2003; de Jong et al., 2004). In addition, a more precise location of the catalytic water was identified and new roles are suggested for $\alpha$ Glu52 beyond that of a general base. The two conformations observed for $\alpha$ Glu52 in the native CaaD structure suggest a role for the side chain in not only activating the catalytic water but in also serving as a gateway for additional waters that replenish the catalytically important water site. Finally, the loss of activity observed for the $\alpha \mathrm{R} 8 \mathrm{~A}$ and $\alpha \mathrm{E} 52 \mathrm{Q}$ mutants of $\mathrm{CaaD}$ is not the consequence of a structure defect.

This research was supported in part by National Institutes of Health Grants GM 65324 (CPW) and P01AI060915 (SDP, ADM) and Department of Defense Grant W81XWH0710445 USAMRAA (ADM). Data were collected on the Southeast Regional Collaborative Access Team (SER-CAT) 22-ID (or 22-BM) beamline at the Advanced Photon Source, Argonne National Laboratory. Supporting institutions may be found at http://www.ser-cat.org/members.html. Use of the Advanced Photon Source was supported by the US Department of Energy, Office of Science, Office of Basic Energy Sciences under Contract No. W-31-109-Eng-38.

\section{References}

Azurmendi, H. F., Wang, S. C., Massiah, M. A., Poelarends, G. J., Whitman, C. P. \& Mildvan, A. S. (2004). Biochemistry, 43, 40824091.

Collaborative Computational Project, Number 4 (1994). Acta Cryst. D50, 760-763.

Emsley, P. \& Cowtan, K. (2004). Acta Cryst. D60, 2126-2132.

Janssen, D. B., Oppentocht, J. E. \& Poelarends, G. J. (2001). Curr. Opin. Biotechnol. 12, 254-258.

Jong, R. M. de, Bazzacco, P., Poelarends, G. J., Johnson, W. H. Jr, Kim, Y. J., Burks, E. A., Serrano, H., Thunnissen, A. M., Whitman, C. P. \& Dijkstra, B. W. (2007). J. Biol. Chem. 282, 2440-2449.

Jong, R. M. de, Brugman, W., Poelarends, G. J., Whitman, C. P. \& Dijkstra, B. W. (2004). J. Biol. Chem. 279, 11546-11552.

Jong, R. M. de \& Dijkstra, B. W. (2003). Curr. Opin. Struct. Biol. 13, $722-730$.

Otwinowski, Z. \& Minor, W. (1997). Methods Enzymol. 276, 307-326.

Poelarends, G. J., Saunier, R. \& Janssen, D. B. (2001). J. Bacteriol. 183, 4269-4277.

Poelarends, G. J., Serrano, H., Person, M. D., Johnson, W. H. Jr, Murzin, A. G. \& Whitman, C. P. (2004). Biochemistry, 43, 759-772. Poelarends, G. J. \& Whitman, C. P. (2004). Bioorg. Chem. 32, 376-392.

Wang, S. C., Person, M. D., Johnson, W. H. Jr \& Whitman, C. P. (2003). Biochemistry, 42, 8762-8773.

Whitman, C. P. (2002). Arch. Biochem. Biophys. 402, 1-13. 